\title{
An Ad Hoc Movement Monitoring Algorithm for Indoor Tracking During Examinations
}

\author{
Sibghatullah H M Sediq ${ }^{a}$ Elisha Tadiwa Nyamasvisva ${ }^{b}$, Atiff Abdalla Mahmoud Arabi ${ }^{c}$, Abudhahir Buhari ${ }^{\text {, }}$ \\ Fares Anwar Salem Hassan ${ }^{\mathrm{e}}$
}

$\mathbf{a}, \mathbf{b}, \mathbf{c}, \mathbf{d}, \mathbf{e}$ Faculty of Engineering, Science and Technology (FEST), Department of Information Technology

Infrastructure University Kuala Lumpur (IUKL)

Kajang, Selangor, Malaysia

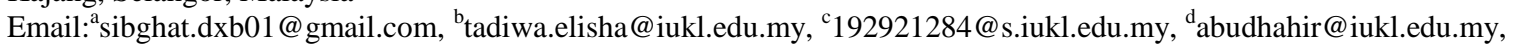

e fares.anwar@iukl.edu.my

Article History: Received: 10 November 2020; Revised 12 January 2021 Accepted: 27 January 2021; Published online: 5 April 2021

\begin{abstract}
Academic dishonesty includes cheating in examinations tests and any academic assignments, plagiarism, fabrication of information or citations, facilitation of acts of academic studies by others, unauthorized possession of examination materials, and tampering with the academic work of other researchers or scholars. These acts can be done before, during, and after the actual examination. This paper proposes a holistic algorithm for setting up an ad-hoc monitoring system to track candidates who leave the examination hall during the examination for either toilet breaks, medical breaks, or otherwise. Since most examinations are held indoors it is especially difficult to use existing GPS and GPRS based methods. We submit a proposal to deploy an indoor monitoring system based on the RSS of carefully placed receivers as they communicate with active tags held by the candidates. This paper outlines an algorithm that is used to place the receivers in such a way as to avoid any blind spots which may be utilized as cheating spots. The algorithm was deployed into a testbed system which was $100 \%$ foul proof. The proposed algorithm can be used to set up the deployment of the monitoring system in any environment where a clearly defined path is identified..
\end{abstract}

Keywords: About four keywords or phrases in alphabetical order, separated by commas

\section{Introduction}

Any developed monitoring system's overarching concept is to allow and empower users to collect data, turn data into information, and regularly distribute information that can be repeated. Monitoring systems enable us to measure the trends of various pointers based on the facts of data collected and analyzed on a given system, event, or set-up in any area of study. A monitoring system is very important to consider new security arrangements and avoid pre-disaster and restoration.

Systematic evaluation and review of after-disaster activities in various fields of monitoring system applications help us monitor the progress and support in assessing the sustainable impact on the affected community[1]-[3]. Therefore, monitoring is to relate the present with the old, the new, and the future. This allows better decision making and avoids potential problems. This paper aims to provide an algorithm for setting up an ad hoc network of sensors to actively collect data necessary for monitoring movements of candidates outside the examination rooms under examination conditions. This will lead to an understanding of the basic ideas behind the exercise of monitoring and evaluation. Reporting will be performed using the methods of instant assessment. The main idea is to prevent cheating before it happens in the exams and limit to the minimum possible, possibilities of cheating especially when candidates take washroom breaks or other forms of breaks which requires them to leave the monitored venue.

\subsection{Why The Need For Monitoring?}

Under the cheating prevention program of the examination, the candidates are assisted in rebuilding their faith and trust, rebuilding value, and facilitating the good manners of the examination in pleasant conditions to strengthen the confidence and respect that an examination deserves. In other words, monitoring ensures that the events are on the correct course by checking them, evaluating progress towards goals, identifying issues as they arise, identifying strengths that can be formed[2], [4]. Monitoring collects information about the access, usage, and satisfaction of the beneficiaries with the process outputs.

\subsection{What is needed for monitoring?}

For the monitoring of exams, an efficient and effective monitoring method is necessary and that method should have the following elements: 
- Information base

- Selection of the activities, outputs, and targets indicators

- Information-gathering tools

- Picking up information

- Information on the process

- Information analysis

\subsection{Where will monitoring take place?}

Continuous Surveillance will be carried out at 3 levels:

- Examination Room

- Washroom

- Transit from examining hall to washrooms

\subsection{When to monitor?}

Monitoring will be conducted regularly and the time and length of monitoring at different levels will be decided upon while planning. Scale and extent of Monitoring are determined by the damage caused by the occurrences being monitored (examinations).

Establishing the monitoring process is done by candidates, the exam department, the surveillance of Invigilators, and the examining board officials involved. The central person in each of the interested parties will be identified; they will take part in this monitoring exercise. Who and who are the participants will be decided upon at the time of observing and organizing.

\subsection{How will monitoring take place?}

A different collection of metrics that have been identified within the system must be evaluated and/or calculated during the entire process (activity metrics, performance metrics, target metrics. Assessments and secondary information will be used to gauge activity metrics regularly and for performance and objective metrics, to select a sample of past reviews endorsed and for which baseline evidence is given, and to evaluate them regularly utilizing relevant reporting tools included in the system work to measure metrics.

\subsection{Review and Evaluation}

The primary goal of the review is to analyze the project more thoroughly than a normal inspection is necessary. The investigation team, with the assistance of partner officers from the advice and counseling team, will conduct frequent formal and in-depth assessments to examine any component of the investigation report[5]-[8].

The goal of the assessment is to evaluate the performance, efficacy, and consequences of these changes and how these changes can be maintained over longer periods in the context of a targeted review of the system. During the assessment, primary success metrics will be identified and evaluated. In other words, an appraisal to see if the specified purpose has been accomplished. After the program, assessments will still be carried out by the reviewing unit, which a more systematic and standardized exercise than those performed during the exams.

\subsection{Monitoring and Management of Examination Risk}

The tracking system is a complex of shifts, threats, and experiences affecting multiple individuals, supervisors (invigilators), and review classes. Danger may be described as probable adverse effects on an exam outcome, exam status or project, or characteristic value occurring in future events. The mixture of chance and impact[6]-[9] is a real risk. Favor is the opportunity and the effect is the permanent change. For eg, one source of risk is the bathroom break time scenario. These threats are created by different sources. However, certain risks are inherent in the practice and certain sums should be treated.

\subsection{Why is monitoring and management of risk is important?}

As the proposed scheme is hoped that it will achieve its targets and objectives, it is important to track and to ensure that the associated potential threats are:

- $\quad$ Stronger and substantial assistance, that is, better service provision for the intended beneficiaries

- Efficient utilization of capital available

- Effective control of future improvements •

- $\quad$ Production, implementation, and maintenance of contingency plans

- $\quad$ Reduce duplication and increase money and time efficiency.

- $\quad$ Strengthened management at all levels by better decision-making

- Greater communication and moderation with other review and collaborators

- Maintain balance time, expenses, and performance. 


\section{2.existing Monitoring Systems}

The Monitoring Information System (MIS) tracking system is specifically connected with the control of main success metrics through priorities and tracking. It can also help to filter relevant decision-making information[10]. A crucial task in the preparation of urgent recovery and recovery programs is to define the regional nature and size of any deception or analyzing time conditions (toilet breaks).

The method of damage assessment and an integrated MIS and GIS system will easily install the device and generate details to prepare and handle the response from the test hall for an alleged cheating situation. These details will help to prepare for the upcoming exams and avoid these related cases a long-term rehabilitation plan. The tracking of the success of post-examination activities would be a core element of information tools[4], [10], [11].

A GIS is capable of generating visual maps based on field knowledge. MIS will be personalized such that harm data are aggregated and maps for surveillance and collaboration [11] are generated. This interconnected framework may be the main component of the monitoring and evaluation system. Based on high-resolution geolocation data, the GIS device damage assessment maps will be developed. This applies where the breaks take place in a different position from the principal place of inspection.

Satellite photographs of high resolution are of immense value for the detection of a real picture of moving objects under surveillance. This is most important in open areas with direct site ties to satellites. Because most exams are performed on-site, this method might not be helpful in our installation.

In movement evaluation, the Global Positioning System ( GPS) plays a very important role. GPS helps to find the location (latitude, longitude, and height) of the impacted objects and other planning and reporting infrastructure with tracking object movements. The GPS coordinates can be conveniently imported into and viewed in maps [5], [11]-[13] into the Geographic Information System ( GIS).

Satellite photographs of high resolution can be easily acquired; time pictures are accessible as a simple image in the archive and are valuable for the duration of the evaluation. However, the use of photographs obtained before an incident involves competent and experienced professionals during the evaluation

In the initial summary test, a variety of generic formats will be used. These forms have been developed to match different kinds of evaluation criteria. It includes material on the physical and human significance and an exhaustive review of activities relating to facilities, claimants, examiners, security, and other applications.

A computerized database management system with the ability to track and report progress on monitoring system results will be created. As the review process develops, this offers a prompt rapid test. The device provides the options online and offline. Data are collected through monitoring agents (active RFID nodes). In the corresponding database enter the info. The website can be reached via Internet connexions [1], [5], [6], [14], [15]. Local area networks are used to send the data to a central server in remote areas. The data submitted to the server interacts with a mechanism that allows both parties to access information. In two steps, the framework is introduced. In the first step, an independent method is developed and tested in the region. A web supporting framework with simple user experience and capability management will be built in the second process.

\section{Proposed system flow}

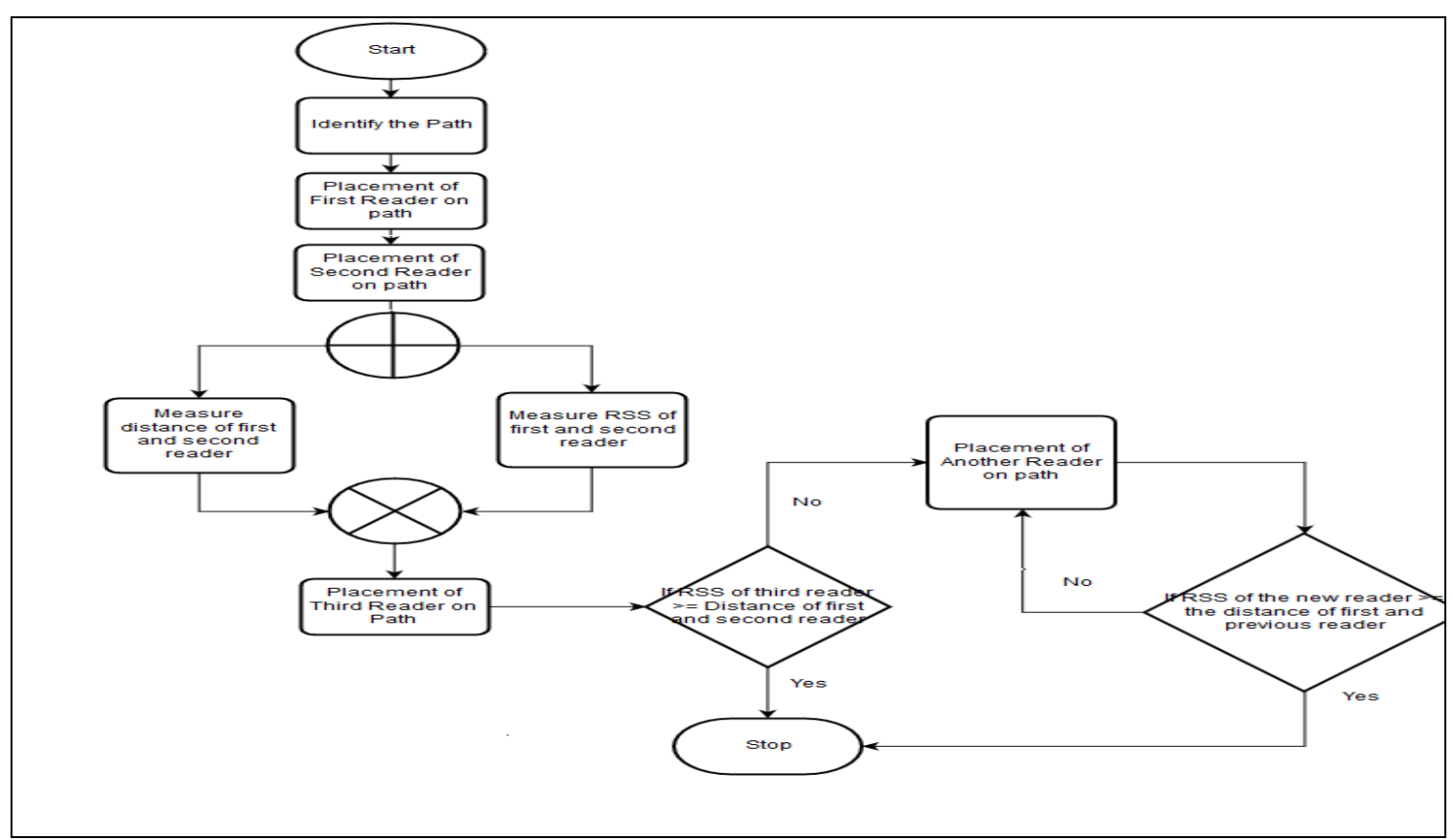

Fig. 1 Proposed System Flow 


\section{1,Big Picture Scenario}

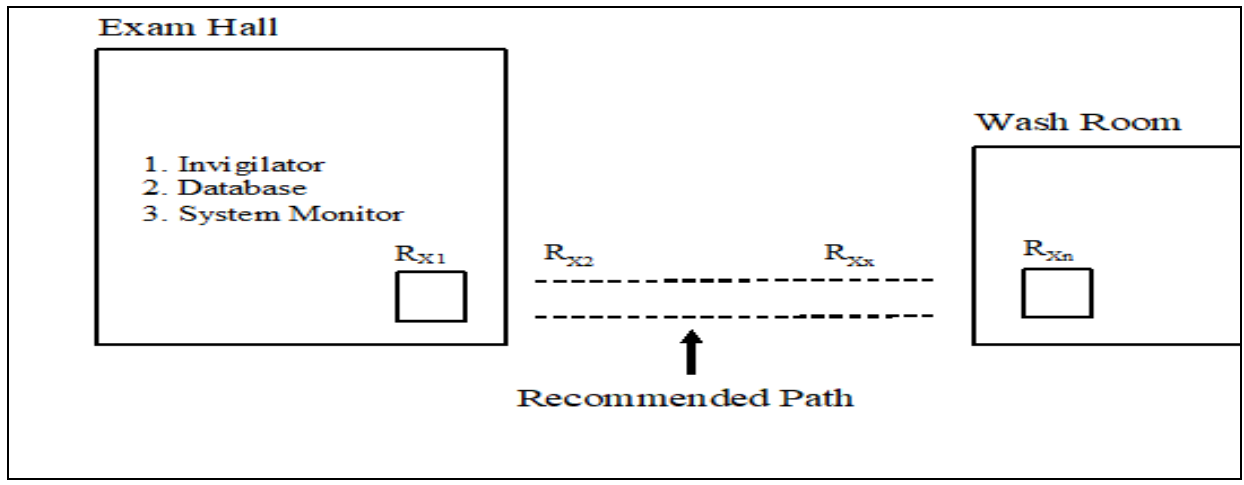

Fig 1: First Scenario of the System

In fig 2, we can see the entire system scenario of how it works and which areas the system operates in. The test hall begins where the student takes the RFID card reader and the student must follow the official prescribed route instructed by the surveillance provider. The exam hall begins. $R x_{1}$ is a passive RFID reader inside the examining hall and all other readers are RFID readers. The $R x_{2}$ is the RFID reader. The last $R x_{x}$ is the $R x_{n}$ which is situated inside the clothing space. $R x_{2}$ is the RFID reader. When returning to the examination hall, the student must take the same prescribed route and return the RFID tag to the surveillance device. The database is used to store the data that has been recovered from active reader signals and to track the RFID identifier.

\subsection{RFID Readers Scenario}

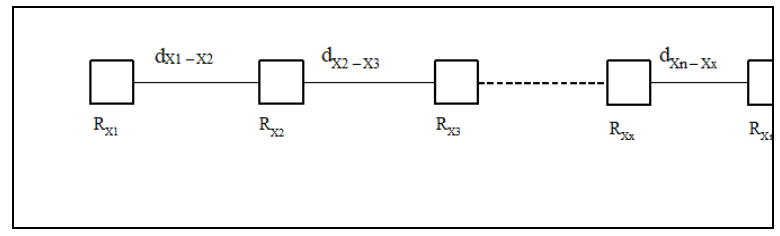

Fig.1: RFID Readers Distance

In Fig. 3. $R_{X 1}-R_{X n}$ are readers' names. $d_{X 1-X 2}$ shows the distance between $R_{X 1}$ and $R_{X 2}$. The first and second readers are $R_{X 1}$ and $R_{X 2}$ and the other readers are passive.

\subsection{Placement of RFID Readers}

The controls (the receivers), or any permitted area outside the monitored room, must be relocated on the predefined route between the examination hall and the washing room. The monitoring agents should often share information on the monitored subjects at a very regular and specific period to gain full autonomy and control of the subjects monitored. Our specified algorithm is original and our work. The algorithm and measurements are described in the following parts.

\subsection{The Algorithm}

If two adjacent control agents are positioned along the route from the examination hall (EH) to the wash-room (WR), the following conditions must be met, as seen in Fig. 4.

Let $R S S_{R x l}$ be the $R_{x l}$ Signal Strength receiver, while $R S S_{R x 2}, R S S_{R X x}$ and $R S S_{R x n}$ are the $R_{x 2}, R_{x x}$, and $R_{x n}$ Signal Strength receiver, of which the first, second, and third are $R_{x l} R_{x 2}, R_{x 3}, R_{x x}$ and $R_{x n}$., receivers and nth and agents of surveillance. It is necessary to remember that in any institution there should be at least three monitoring officers:

$\mathrm{R}_{\mathrm{x} 1}$ is identified as a passive transceiver in the examination hall that starts monitoring and verifying that monitoring is terminated.

It must be positioned in a predefined direction such that $R S S_{R x x}$ is the second last monitoring agent to be positionedwhere $R_{x n}$ is the last monitoring agent.

If $R S S_{R x x} \geq R S S_{R x I}$ the path of the three agents $R_{x l}, R_{x x}$, and $R_{x n}$ will be tracked absolutely. The condition as seen in the diagram. Four lower down 


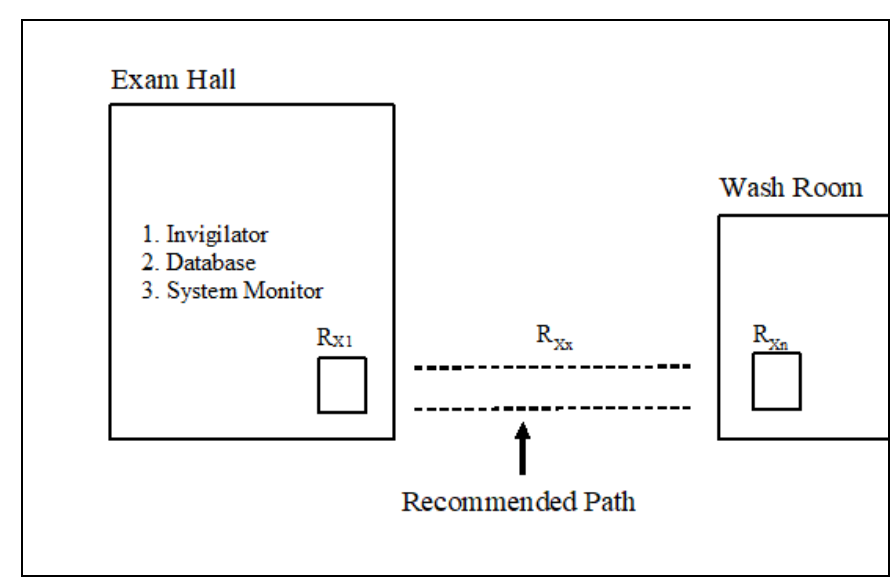

Fig. 1: 3-RFID Reader placements

Should $R S S_{R x x} \leq R S S_{R x l}$, an additional monitoring agent $R x_{2}$ between $R x_{x}$ and $R x_{1}$ will be created. $R S S_{R x l}$ and $R S S_{R x 2}$ are evaluated under the same state once again. If $R S S_{R x 2} \geq R S S_{R x x}$, then the four $R x_{1}, R x_{2}, R x_{x}$, and $R x_{n}$ agents are completely monitoring the route. The condition is seen in Fig. 5.

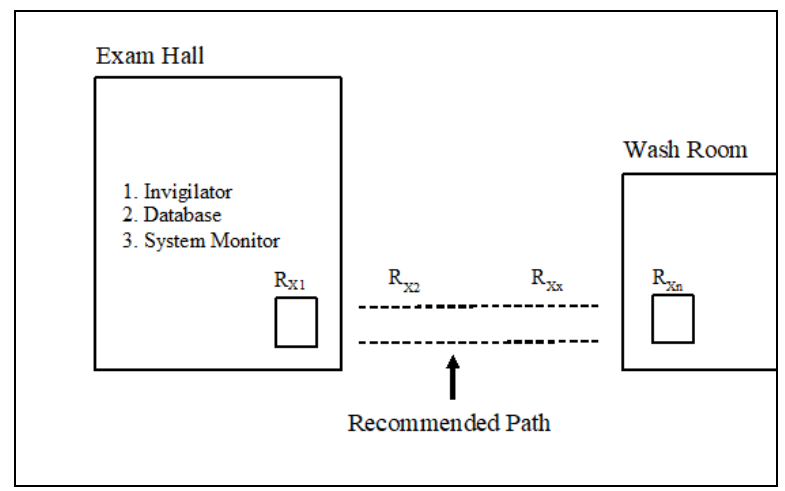

Fig. 2: 4-RFID Reader placements

Should $R S S_{R x 2} \leq R S S_{R x l}$, a separate control agent between $R x_{x}$ and $R x_{1}$ would be set up. The name of the tracking agents will be changed to 1 (one) location for an $R x_{l}$ agent. For example, $\mathrm{Rx}_{2}$ will be called $R x_{3}, R x_{3}$, and $R x_{4}$, etc. when $R x_{2}$ is to be checked for $R S S_{R x l}$ and $R S S_{R x 2}$ again in the same state. The checking is carried out before complete coverage by the Monitoring Agents of the entire recommended route. The full path recognition and modeling algorithm are given below.

Predefine a recommended path between the examination room and washroom (Path)

Place Rxl at the examination venue as the first receiver

Place Rxn at the washroom as the last receiver

Check $R S S_{(\max )}$ for $R x_{1}$ and $R x_{n}$

Measure $D_{(R x I-R x n)}$ the distance between $R x_{1}$ and $R x_{n}$

Place Rx $x_{x}$ on Path $(R x I-R x n)$ such that $R S S_{R x x} \geq R S S_{R x n}$

Measure $D_{(R x x-R x n)}$ the distance between $R x_{x}$ and $R x_{n}$

Measure $D_{(R x x-R x x)}$ the distance between $R x_{x}$ and $R x_{I}$

If

$D_{(R x x-R x l)}$ is covered fully by $R S S_{R x x}$ then

Stop

Else

Place $R x_{2}$ on Path such that $R S S R x 2 \geq R S S_{R x x}$

Measure $D_{(R x x-R x 2)}$ the distance between $R x_{x}$ and $R x_{2}$

Measure $D\left(R x_{2}-R x_{1}\right)$ the distance between $R x_{2}$ and $R x_{1}$

If

$\left(R x_{1}\right)$ is covered fully by $R S S_{R x 2}$ then

Stop 
else

While $\left(D_{(R x 2-R x 1)}\right)$ is not covered fully by $\left.R S S_{R \times 2}\right)$

Rename $R x_{2}$ to $R x_{3}$ (incremental)

Place $R x_{2}$ on Path such that $R S S_{R x 2} \geq R S S_{R x 3}$

Measure $D_{(R x 3-R \times 2)}$ the distance between $R x_{3}$ and $R x_{2}$

Measure $D_{(R x 2-R x I)}$ the distance between $R x_{2}$ and $R x_{1}$

If

$D_{(R x 2-R x I)}$ is not covered fully by $R S S_{R x 2}$

loop

Else

Stop

End

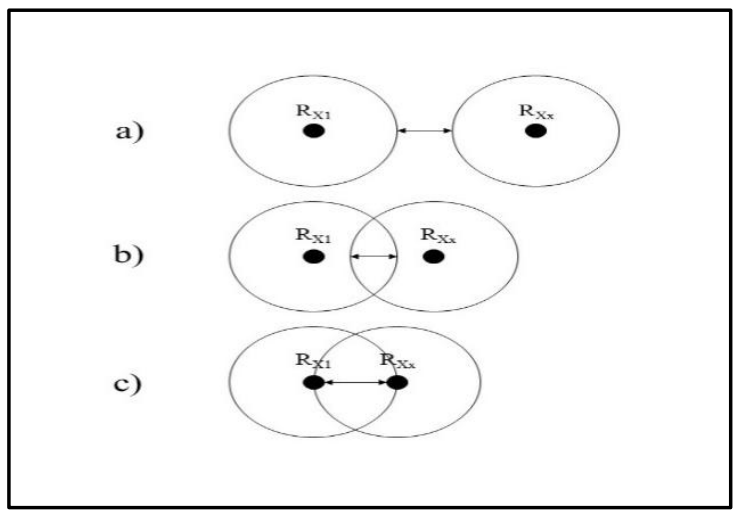

Fig .3: Placement of RFID Readers

In fig. 6 above. We are faced with three distinct situations, a, b and c. In Scenario a, the $R S S$ of the $R_{X I}$, which makes this the system's worst scenario, cannot meet the RSS of the $R_{X 2}$.

In scenario b, the RSS for the two readers' overlaps, but the issue is that the reader's $R_{X I}$ coverage area will only hit $R_{X 2}$ and in scenario c, the $R S S$ of the $R_{X 1}$ reader reaches or equal to $R_{X 2}$ is the optimal placement. We can also say that the difference between the two readers can be described by $d_{R X 1-R X 2}: X_{1}>=X_{2}$

\section{Conclusion}

This paper has implemented a new simple but powerful algorithm for putting receivers on a pre-identified monitoring path. The machine uses the signal intensity obtained by the subject to constantly pick up an activated card when traveling to and from the unmonitored inspection regions. In such cases, the current GPS and MIS networks cannot run precisely inside or multi-layer structures, since they are not capable of operating precisely

\section{References}

A. Kessler and J. Tanburn, "Why Evaluations Fail : The Importance of Good Monitoring," no. August, pp. 1-9, 2014.

F. A. O. Otieno, "The Roles of Monitoring and Evaluation in Projects."

K. Hobson, R. Mayne, and J. Hamilton, “A step by step guide to Monitoring and Evaluation,” Evaloc, pp. 1-60, 2014.

R. K. Gupta, S. Dhaulta, R. Kumar, M. Balasubramaniam, G. Münzenberg, and W. Scheid, "Closed-shell effects from the stability and instability of nuclei against cluster decays in the mass regions 130-158 and 180-198," Phys. Rev. C - Nucl. Phys., vol. 68, no. 3, p. 10, 2003.

S. Paulo, C. Shewbridge, D. Nusche, and H.-D. Herzog, "Evaluation and an Assessment Frameworks for Improving School Outcomes Common Policy Challenges," Educ. Train. Policy, vol. 21, 2009.

M. S. Devi and H. Mittal, "Review of Computerized Evaluation Tools in Education,” Int. J. Artif. Intell. Comput. Res., vol. 4, no. 2, pp. 111-117, 2012. 
S. Practice, "2020 Risk Monitoring and Examination Priorities Letter," no. January 2020.

H. Items, S. P. Risks, O. Risks, M. Risks, and F. Risks, "2019 Risk Monitoring and Examination Priorities Letter," no. January 2019.

R. S. Pressman, S. Engineering, and W. Lord, "Risk Mitigation, Monitoring and Management Plan," pp. 1-21.

A. Berisha - Shaqiri, "Management Information System and Decision-Making," Acad. J. Interdiscip. Stud., no. December 2014.

K. Chang, "Introduction to Geographic Information Systems," no. August 2012.

T. M. L. B.; S. A. M.; C. Wellford, "Document Title: Global Positioning System (GPS) Technology for Community Supervision: Lessons Learned," Cent. Crim. Justice Technology, 2007.

I. Teaching and L. Program, "GPS Technology Introduction : Background :" 2007.

S. Heckley et al., "Why is monitoring and evaluation a challenge in sustainable urban mobility planning ?," no. January, p. 9, 2014.

D. A. chatterjee Dr.Asim chatterjee, "Present Evaluation Method of Examination: A Critical Survey," IOSR J. Humanit. Soc. Sci., vol. 11, no. 1, pp. 45-49, 2013. 